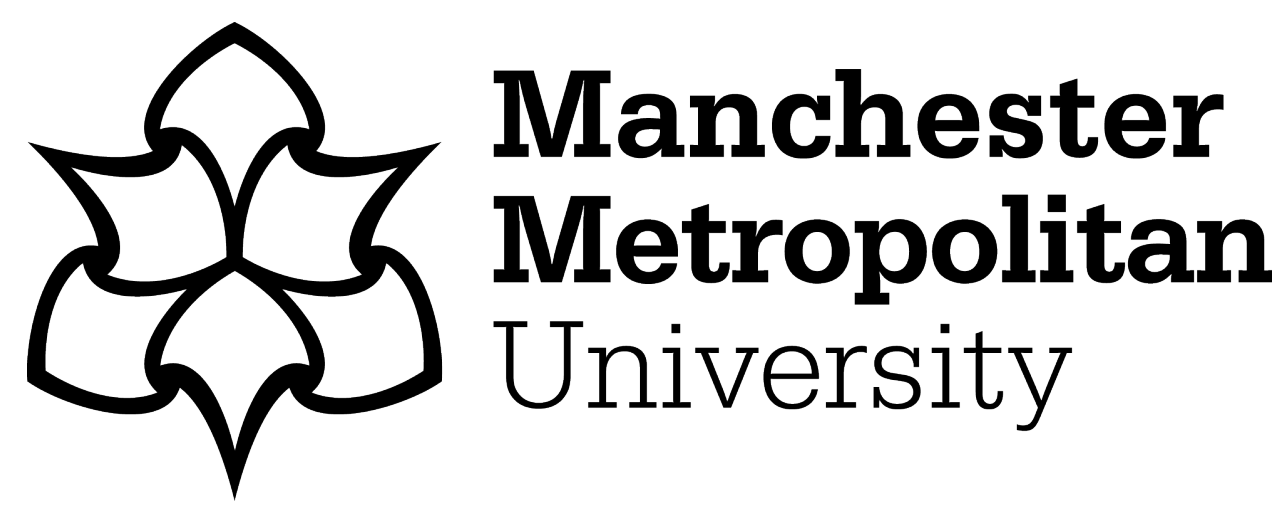

Davies, K, Goldbart, J, Marshall, J and Brown, L (2017) Co-working: parents' conceptions of roles in supporting their children's speech and language development. Child Language Teaching \& Therapy, 33 (2). pp. 171-185. ISSN 0265-6590

Downloaded from: https://e-space.mmu.ac.uk/617512/

Version: Accepted Version

Publisher: Sage Publications Ltd.

DOI: https://doi.org/10.1177/0265659016671169

Please cite the published version 


\section{Co-working: parents' conception of roles in supporting their children's speech and language development}

Karen E. Davies, Faculty of Biology, Medicine and Health University of Manchester

Julie Marshall, Faculty of Health, Psychology \& Social Care, Manchester Metropolitan

University

Laura J.E. Brown, Faculty of Medical and Human Sciences, University of Manchester

Juliet Goldbart, Faculty of Health, Psychology \& Social Care, Manchester Metropolitan

University

Funded as part of NIHR Programme Grant, Child Talks What Works 
Abstract

Background: Speech and language therapists' (SLTs) roles include enabling parents to provide intervention. We know little about how parents understand their role during speech and language intervention or whether these change during involvement with SLTs. The theory of conceptual change, applied to parents as adult learners, is used as a framework for understanding changes in parents' conception of roles during intervention.

Aims: The aims of the study were (i) to investigate parents' conception of their own and SLT roles during speech and language therapy intervention (ii) to explore changes in parents' conception of their role as they work with SLTs.

Method: A qualitative study was conducted using semi-structured interviews with 14 parents of pre-school children with speech and language needs referred to speech and language therapy in the UK. A subset of 8 parents participated in a longitudinal study over nine months to track any changes in conception of roles during intervention. Interviews were analysed using Thematic Network Analysis to identify basic, organising and global themes. Framework Analysis was used to compare participants over time and identify any changes reported by parents. 
Results: Parents had a firm conception of their role as advocates but did not express a clear notion of their role as intervener before involvement with the SLT. During intervention, some described changing their conception of role including adopting roles as 'implementer' and 'adaptor' of intervention. In some cases, parents described changes in their approach to parenting which they associated with adopting more active roles as interveners.

Conclusions: Policy statements about parental choice and co-working typically present parents' conception of roles as fixed. This research shows that parents may change their conception of role as they work with SLTs and become increasingly involved as interveners. Implications for practice include negotiating roles in the partnership before intervention begins and enabling parents to adopt an intervener role.

Key words: parents, conception of role, speech and language therapy, intervention.

\section{Introduction}

Policy and practice in education, health and social care in the UK has emphasised the benefits of parents as partners in supporting children's learning (Department for Education and Department for Health, 2015; Goodhall and Montgomery 2014; 
Goodhall and Vorhaus, 2011). Speech and language therapists (SLT) and parents form a partnership with the intention of improving pre-school children's speech and language development. There is, however, only limited research on parents' conception of their roles and their expectations of involvement in supporting their child's speech and language development. It is important to understand how parents perceive their role, and consider how to build parental confidence in adopting an active role during intervention. There are a number of theories that consider adult behaviour as part of the context for children's speech and language development, such as the International Classification of Functioning, Disability and Health - Children and Youth (ICF-CY) and Bioecological Model of Development (WHO 2007; Bronfenbrenner and Morris 2006) . This study uses the theoretical perspective of 'Conceptual Change' (Vosniadou 2013) to identify qualitatively distinct ways in which parents conceptualise their role and to capture the way in which these conceptions may change. The theory suggests that conceptual change occurs when an individual finds their existing conception is inadequate and encounters new conceptions that appear plausible and potentially valuable, leading to restructuring of prior knowledge (Vosniadou, 2013). Consequently, the conflict between existing understanding and new knowledge triggers a significant shift in an individual's understanding (Pintrich 
et al., 1993; Kaufman et al., 2013; Vosniadou 2007). Conceptual change has been used to understand young people's learning but, as yet, has not been widely explored in the context of adult learning, particularly in relation to people's understanding of health (Kaufman et al., 2013). Further, conceptual change theory is not widely used in the discipline of speech and language therapy.

This paper proposes that conceptual change theory could offer a new and useful framework to understand changes that occur in parental understanding during partnership practice between SLTs and parents. In particular, the process of 'collaborative reflection' prompting conceptual change (Miyake 2013, p.469), could have a useful application to the way parents and SLTs approach problem solving as a joint or shared endeavour prompted by questions between participants. For instance, roles are exchanged between 'task doer' and 'monitor' during learning, as part of an iterative cycle of understanding leading to conceptual change.

Following the convention in 'conceptual change' this paper refers to conceptions as different ways of understanding, in contrast to perceptions that refer to processing immediate information. Conceptual frameworks are often well established, based on learning over time, and consequently may be resistant to change. Clarifying the possibility of change in parents' conception of their roles should foster a better 
understanding of how to support parents' learning during partnership with SLTs. The current study complements studies that theorise the influence of parents on their child's speech and language development as part of the interaction between biological and environmental factors, such as the Bioecological Model of Development (Bronfenbrenner and Morris 2006; Cunningham and Rosenbaum, 2015).

Although little is known about parents' conceptions of their roles, there is evidence to suggest that when a child is referred for a speech and language assessment, parents assume an advocacy role for their child, identifying concerns, seeking advice and judging progress (Glogowska and Campbell, 2004; Law and Roy, 2008; Lindsay and Dockrell, 2004; McAllister et al., 2011). Many parents appear to assume this role with confidence as illustrated by decision-making about whether to follow up referral to speech and language therapy. In a survey of 109 parents of pre-school children with speech sound difficulties in Australia (McAllister et al., 2011), two thirds of parents had not sought services and half of these made decisions that services were not needed. In the UK, findings from a qualitative study of twenty parents (Glogowska and Campbell, 2004) reported that they controlled when a referral to the SLT occurred. Such decision-making indicates that parents are advocating on their child's behalf, 
based on some form of evaluating their child's difficulties and making a judgement about when to seek help.

Relatively few studies have investigated parents' conception of role once their child is receiving intervention from the SLT. There are indications that parents feel uncertain about their role, considering the SLT as 'fixer' (Carroll, 2010) or expressing concern about 'interfering' with the work of the SLT (Watts-Pappas et al., 2016). Parents reported adopting an 'observation' role during SLT-led intervention and 'implementer' role in doing homework assigned by the SLT (Watts-Pappas et al., 2016).

Nevertheless, previous research suggests that parents may be as effective in administering intervention as clinicians (Law et al., 2008) and can be taught strategies to support children's speech and language development (Roberts and Kaiser, 2011). This raises a question about how much SLTs expect and enable parents to adopt roles that involve more than observing or implementing homework. In a study investigating 67 parents' views of intervention, only $10 \%$ of parents' comments referred to SLTs supporting parental involvement (Washington et al., 2012). This suggests that parent participation may not be an explicit priority in the delivery of usual clinical practice, with both parents and SLTs tending to perceive SLTs as the intervener. Even in the 
context of parent centred intervention, such as parent-child interaction, SLTs considered that parents find it hard to reflect on their interaction with their child, and that goal setting remains SLT-led (Klatte and Roulstone, 2016).

The literature presents a confused picture of the roles that SLTs assign to parents, either explicitly or implicitly by their own expectations of parents or style of service delivery. SLTs regard parents as the main mediator of change for young children (Roulstone et al., 2012) with 'homework' forming a regular part of intervention (Watts-Pappas et al., 2008). However, SLTs value 'direct' intervention, delivered by the SLT to a child, more highly than 'indirect' approaches delivered by others (Pring et al., 2012). This suggests that SLTs may regard parent-led intervention as less valuable than therapist-led intervention.

Traditional models of speech and language therapy practice, based on parents as 'implementers' of therapist-directed activities, may reinforce the roles parents adopt (Watts-Pappas et al., 2008). SLTs may be unaware of how they influence parents' roles, believing parents are included as co-workers, whilst maintaining SLT-led approaches. Further evidence indicates that SLTs did not acknowledge or build on parents' existing approaches to promoting speech and language development 
(Marshall et al.'s, 2007) suggesting that therapists' behaviour may reinforce expectations that SLTs assume the intervener role.

Enabling parents to adopt different conceptions of their roles during intervention could create opportunities for parents to assume more clearly defined roles in the partnership with SLTs. To date, few studies have investigated parents' understanding of involvement in intervention, with a gap in evidence regarding parents' conception of roles. The current study aimed to identify parents' conception of roles and to track changes in conception as they participated in typical speech and language therapy intervention provided by services in the UK. The study posed the following research questions:

1. What are parents' conceptions of their own and SLTs' roles in speech and language therapy intervention for their pre-school children?

2. How do parents' conceptions of these roles change during speech and language therapy intervention?

\section{Method}

1. Study design 
This paper reports part of a larger investigation of both parent and SLT conceptions of their own and each other's roles. It presents the findings of a qualitative study using a cross-sectional and longitudinal design, to collect data from participants during typical management by SLTs in the UK. Data were collected between January 2011 and March 2013.

In line with the British Psychological Society's ethical principles, all participants provided written informed consent and were assured their data would be anonymous. Data were stored securely according to university procedures. Ethical approval was given by Manchester Metropolitan University Ethics Committee and NHS Research Authority (NRES Committee North East).

\section{Participants}

Inclusion criteria for the study were parents with (i) children referred for assessment of primary speech and language needs; (ii) children aged 2.00-5.11 years. In the case of a child attending with both mother and father, the invitation to participate was issued to both. Exclusion criteria were those children with neuro-developmental difficulties or marked social need. Twenty parents were invited to participate and fourteen were recruited to the study. Two mother and father pairs were interviewed 
together and analysed as one set of views as, in both instances, the father answered the questions. A smaller subset of eight parents participated in the longitudinal study. The parent participants were from low and medium SES groups, as indicated by educational level, employment and home postcode (Table 1). Reasons for referral provided by the referrer were predominantly described as language or speech delay (Table 2).

Speech and language therapy managers from four NHS sites in England were approached to act as gatekeepers to facilitate recruitment to the study. Managers identified SLTs working with pre-school children, who were then invited to participate in the study (12 SLTs). They invited parents of children scheduled for first assessments to participate. The study used purposive sampling to collect data from a range of services delivering different models of provision and from areas with different demographics. Parents were recruited to the study incrementally over 6 months and, in keeping with qualitative methods, the sample size was determined by the achievement of theoretical saturation, with recruitment completed when no new themes were generated from additional data (Richie et al., 2013). Recruitment took place on-site where parents had opted to attend their first appointment. Table 1 placed here 


\section{Table 2 placed here}

\section{Procedure}

a. Data collection. All participants were interviewed initially using a semistructured format, with open ended questions that allowed parents to give their opinions in detail, explaining and elaborating as the interview progressed. Interviews are an ideal method for exploring people's perceptions of complex constructs, such as conceptions of roles. The informal format of a semi-structured interview allows the researcher to pursue lines of inquiry whilst maintaining a systematic approach to data collection (Breakwell, 2006).

Three interviews with each parent were planned over a 30 week period whilst they received typical care according to the model agreed by the service. This could be direct therapy with the SLT, usually in weekly appointments over 4-6 weeks, parent groups, sample sessions modelling intervention, written programmes, review or discharge. Each interview followed a topic guide (Appendix 1), prepared and piloted with the assistance of a parent reference group of eight parents. Each interview explored parents' views of their child's progress, accessing support, perception of their role, and changes in role, during involvement in speech and language therapy. The topic guide for the first set of 
interviews consisted of nine open ended questions. Subsequent interviews included questions on support, decision-making and expectations.

Interviews took place in routine settings such as clinics, children's centres and participants' homes after initial assessment with SLTs. The interviews were scheduled to last 20-30 minutes. Parents were invited by the researcher to participate in two further interviews, approximately 10 and 30 weeks after the first interview (Table 3). Subsequent interviews were either face to face or telephone interviews, depending on the preference of the participants.

Interviews were audio recorded, transcribed verbatim and uploaded onto NVIVO (2012). NVIVO is a software tool for organising qualitative data and supporting systematic analysis of research findings. Contemporaneous field notes were also made to record initial ideas and issues, contributing to the analysis of each case.

Table 3 placed here

The researcher (KD) was an SLT and able to benefit from understanding the professional context and respond flexibly to recruiting parents in a variety of service settings. The risks associated with researcher bias were countered by using transparency of research design, reflexive commentary and regular input from an advisory group and supervisory team. 
b. Data analysis. The analysis involved a two-stage process: the first stage interrogated the data from the first set of interviews, identifying themes using Thematic Network Analysis (Attride-Stirling, 2001). The second stage analysis compared the longitudinal data from each participant, and between participants, using Framework Analysis (Ritchie and Spencer, 2002) from the first, second and third set of interviews. Each transcript was analysed as soon as the data were collected so that codes were defined and systematically recorded incrementally. Initially, one researcher (KD) coded interview transcripts from participants inductively. Codes, themes and networks were discussed and agreed by the research team (LB, JG, JM) to achieve an accurate analysis and interpretation of data and contribute to the rigour of the analysis.

Codes were categorised into descriptive themes initially and then re-categorised into broader themes. Overarching themes were identified and a hierarchical network of basic, organising and global themes was created according to Thematic Network Analysis. For example, the global theme of 'parents' conception of role' had three organising themes of 'advocate', 'intervener' and 'taking responsibility'. A full list of codes is available from the authors. The relationships between themes were explored and were used to support the interpretation of the findings. 
Themes from the first stage analysis provided a priori themes for the development of the framework used in stage two to compare cases and track changes over time for each individual case.

\section{Results}

The results from the first set of interviews (14 parents) generated the global themes of parents' conception of their own role and SLTs' roles, whilst results from the second and third set of interviews ( 8 and 5 parents respectively) revealed the nature and extent of changes in parents' conception of role. Parents' own words are used to illustrate the findings, marked as P1-P14 to denote individual parents.

Parents' conception of their role during involvement in speech and language therapy

The data from the first set of interviews revealed a global theme of 'parents' conception of roles'. Within this theme there were organising themes of advocate, intervener and taking responsibility for supporting their child's speech language development (Table 4).

Table 4 placed here 
a. Advocate role. All parents interviewed talked clearly about their advocacy role, seeking advice and support for their child. This was expressed as a response to making a judgment that their child's speech and language development was not progressing in line with their own and others' expectations. Often this involved comparing their child with others in their social network. Some parents recalled being prompted by another person, such as a teacher or family member. All the parents expressed a willingness to be involved in speech and language therapy, in order to support their children, despite considerable uncertainty about what would be expected of them. For some parents, this generated an anxiety about involvement. However, in spite of expressing feelings of anxiety, many parents recounted persistence and tenacity in seeking advice.

Their views fitted into three basic themes: (i) responding to the concern of others (ii) raising concerns themselves and (iii) actively pursuing advice, including judging the value of advice received from others. Those who responded to the concern of others conveyed relatively little participation in the decision about referral, but accepted other people's concern, in spite of their own doubts about the importance of following up the referral: 
We went to the health visitor and she asked how many words she says and I say 'no she doesn't' and then she said, we need to refer her (P13)

The second basic theme, raising concerns, included parents who expressed their advocacy through alerting others to their observations of their child. This was often linked to parents' anxiety about whether their child was 'developing normally' (parent's words). They perceived their role as negotiating access to appropriate help, often in the face of resistance from others:

I did have concerns. The health visitor didn't, but I did. (P3)

They discussed their anxieties with nursery teachers, health visitors and family members, as a process of raising concerns, linking this with taking responsibility for initiating a referral to speech and language therapy.

The third basic theme expressed advocacy as pursuing advice, including judging the quality of advice they received. Parents used words such as having to press and push, often seeking advice from a variety of professionals who did not necessarily understand the parents' concern, as illustrated by the following quote:

I had to mither quite a bit at school, cos it was nursery, they said, oh she plays and I said it's not about her playing, it's her talking (P2) 
As parents pursued support they indicated that they evaluated the quality of the advice provided, specifically seeking advice from an informed and experienced professional:

I think to come and get a proper opinion like this has helped me a lot, rather than listening to someone else (P4)

b. Intervener role. In contrast to their description of their advocacy role, parents voiced uncertainty about their role in helping their child's speech and language development. However, the majority expressed a desire to learn, and adopt a role as an intervener, of some sort. Parents' views indicated three basic themes associated with the intervener role: (i) attender (attending appointments) (ii) implementer (doing activities as prescribed by SLT) and (iii) adaptor (adapting approach to communication and interaction in the home).

Parents who tended to perceive their role as 'attenders' felt uncertain about how to support speech and language development themselves and were therefore keen for their child to be seen frequently by a professional who understood speech and language development. This implied that a conception of 'attender' might be rooted in parents' lack of knowledge, experience or self-efficacy in relation to enabling speech 
and language development, rather than an unwillingness to devote time to supporting their child or expecting SLTs to find a solution.

The second conception of the intervener role described by parents was an 'implementer' or 'helper' role. This was characterised as doing as they were asked by the SLT. Their words suggest a confidence in implementing prescribed activities, but little indication of adapting activities or incorporating advice into routines at home:

They have given a little bit of homework, things we can do to help and anything they suggest we do, we're going to make sure we do the best we can (P11)

The third conception of the intervener role was expressed as 'adaptor', referring to changing approaches, adapting circumstances and teaching their child, rather than implementing prescribed activities only. One parent commented:

I think we'll be taking more of an active role rather than just looking at pictures. Doing activities and talking through them so you can incorporate them in your normal life (P9)

Several parents described a point of realisation that interaction with their child needed to be different, suggesting a marked change in their thinking. P1 explicitly commented that her current manner had not helped her child's communication: 
Got to back off with the pressure, not so pressurising just because you want him to' and then 'More about having fun, if he's having fun he more happily talks (P1)

c. Taking responsibility. The balance of responsibility for supporting a child with speech and language needs depends on the roles adopted by parents and professionals during intervention. There were three basic themes indicating parents' conceptions of their role in taking responsibility: (i) expecting SLTs to lead intervention, (ii) implementing intervention themselves and (iii) influencing the design of the intervention. There were no examples of parents who assumed that SLTs should take full responsibility for intervention. Some parents expected SLTs to provide intervention, whilst others expressed a readiness to share responsibility, even when they were uncertain about the best course of action. Some parents expressed ambivalence regarding who should assume the lead responsibility. P11 initially expected the SLT to take responsibility for the intervention, but later in the same interview articulated changes in his conception of role:

It would have been nice to have a proper one on one session with (child)..... It's better off where we're taught ourselves and we're doing it ourselves constantly at home, which I totally agree with (P11) 
A small number of parents explicitly expressed a view of themselves as taking responsibility for intervention from the outset:

It's something I'm doing with the boys as well, I'm helping them and not just letting someone else do the work (P3)

Finally, some parents in the study were keen to influence the decision-making regarding what happened in the intervention and how it was delivered. This related to practical aspects of intervention such as whether they had further intervention from the SLT, when or where this might take place, or how to incorporate activities in busy family schedules:

The choice was open to me, if I wanted to take the sessions or if I just wanted to leave it or just take their advice, that was down to myself, there was no pressure. (P9)

In summary, parents commented that they had little knowledge or experience of language development to draw on and described themselves as new to thinking about helping their child's speech and language development. These data suggest that parents' role conception, in relation to supporting their child's speech and language development, is tentative and open to change, but also reveals different levels of involvement. For example, within the advocacy role, parents showed a tendency to 
one of three basic themes, 'responding to other's concerns', 'raising concern', and 'pursuing advice/judging advice', reflecting levels of involvement and readiness to take an active part.

Parents' conception of SLTS' role during intervention

The global theme of 'parents' expectation of support' consisted of the organising themes of 'expectation of the SLT roles' and 'expectation of the speech and language therapy procedure'. This paper reports parents' expectation of support. Parents' conception of SLTs' roles consisted of four basic themes identified in the first set of interviews as roles of assessor, intervener, intervention planner and teacher (Table 5). However, their conceptions of the SLT role were vaguely formulated and they expressed uncertainty of what speech and language therapy would involve.

\section{Table 5 placed here}

a. $\quad$ SLT as assessor. Parents were seeking an informed opinion about their child's development, anticipating that the SLT would 'check' how their child's speech and language was progressing. This was accompanied by a desire to know how their child's progress compared with others. Parents were uncertain about making 
judgements about their child's developmental progress, illustrated by one parent saying she (herself) was 'not very good at assessment of her.'

b. SLT as intervener. A number of parents expressed an expectation that the SLT would 'do' the intervention directly with their child, with comments such as:

He needs to see a therapist possibly on a weekly basis. (P11)

Parents' accounts did not explicitly indicate the rationale for expecting SLTs to do the intervention, but they referred to their perception of SLT expertise, knowledge and experience as important factors in determining their views, contrasted with their own inexperience. As one parent (P14) expressed, 'that's her expertise; it's not mine'. There were indications that this conception may have prompted some parents to doubt the benefit of courses for parents, believing that their child's difficulties should be addressed directly:

The course is for me rather than him, so in regards to speech, I'd have liked something for him. (P6)

c. SLT as intervention planner. Parents expressed a conception of the SLT as planning and providing activities to help their child's speech and language development. One parent believed she was 'being led,' whilst another was more direct in expecting the SLT to tell her what activities she should do. There was evidence of a 
relationship between parents' uncertainty about what to do themselves and expecting to be given a plan or advice from a knowledgeable individual, as illustrated by this parent:

Sometimes as a parent you don't know what strategy to follow and what's going to work and obviously they're more experienced, and sort of feel they can advise уои. (P3)

d. SLT as teacher. Parents' regularly referred to expecting the SLT to teach them how to help their child. Many parents used phrases such as being 'shown what to do' and 'given techniques'. A number expressed an explicit preference for the SLT to teach them in order to learn new skills, 'I want someone else to teach me the techniques' (P1). This parent expressed a hope that learning would prompt a significant change in her practice:

I think they will just show me how to get things out of him, cos the way I'm trying at the moment is obviously not working-hopefully show me new ways (P1)

Changes in parents' conception of their own role during intervention

Results from the second and third set of interviews with eight and five parents respectively suggested that, during involvement with the SLT, parents gained 
knowledge, learnt new activities and techniques, and considered themselves as facilitators of speech and language development. Some accounts indicated that parents believed their role changed during their child's involvement in speech and language therapy. Parents showed inclinations to one of three groups, although they did not present their conception of roles as fixed and rigid.

a. Conception of role remains unchanged. Some parents reported that their knowledge of how to help speech and language development had increased following the SLT assessment, but described little change in their behaviour over the successive weeks. For example, one parent believed the SLT had given her appropriate information but felt unable to become an intervener. She cited time, skills and inclination as limiting factors. She exemplified this by referring to her approach in another area of parenting, which illustrated her preference for others to teach her child:

Sometimes I feel I expect other people to do it, taking her to French classes, I sit back and let her do it with the teacher rather than me (P12, Second interview) 
She explicitly linked this expectation to doubts about her own ability to provide support, referring to the tension between competing demands of family and home, constraining her time and motivation.

b. Gaining a conception of role as an 'implementer'. A number of parents described adopting an implementer role. They perceived their role as 'doing' activities as directed by the SLT, with little reference to changing communication generally, or integrating activities into day to day interaction with their child. For example, one parent explained:

They have given a little bit of homework, things we can do to help and anything they suggest we do (P11, first interview)

Some parents' accounts indicated a degree of uncertainty and inconsistency in the perception of their role as an implementer, on the one hand anticipating that they will do as advised by the SLT and on the other, believing that their role does not need to change, as illustrated in the following exchange,

P5: It's going to help me help him.....telling me what to do

I: How is that going to be different to what you're doing now?

P5: it's not, cos what she's told me to do, I'm doing already 


\section{I: so you're not looking for anything extra?}

P5: um... no not really

Inconsistencies in parents' responses could be interpreted as indicating that their conception of role was unclear. However, it may also indicate that conceptions were emerging as understanding of their roles changed.

c. Gaining a conception of role as an 'intervener'. A number of parents in the study described changes in their conception of role as learning to adapt their interaction and activities to enable their child's speech and language to develop in the home context. Their accounts conveyed thinking differently about how to support their child's learning, as well as what they should be doing as homework tasks. For example, one parent explained that she learnt techniques, but also 'completely changed' her interaction with her child, expressed as a transformation in her thinking and behaviour:

I was more like a master...I was constantly battling with him, shouting at him, trying to confine him, but now I'm more like a parent...I'm more of his loving parent (P1, third interview) 
Another parent described adopting more positive approaches to parenting her child, associated with working with the SLT:

More positive...more in control of things, I'm helping more, controlling it more (P3, third interview)

Some parents regarded their involvement in speech and language therapy as a learning process, adding to their understanding of their own behaviour in relation to their child's language learning. P4 explained:

She gave us so much advice and she was telling us that everything we were doing was right....loads of advice, it was a good experience and we learnt a lot'. (P4 third interview)

Part of the learning process included reflection on their own communicative behaviour with their child. For example, P7 found she learnt from other parents as part of a parent group, P1 and P14 referred to observing how SLTs worked with their children and seeing their children respond differently, prompting changes in their own approach. P3 described learning as she did activities with her children. The evidence does not reveal whether SLTs explicitly intended to promote changes in 
parents' role conception as part of the intervention, or whether such changes were an outcome of addressing children's speech and language difficulties.

\section{Discussion and implications}

This paper reports the findings of an exploratory study of parents' conceptions of their own and SLTs' roles, and how these conceptions may change, during involvement in intervention. Two main findings are discussed (i) parents have a firm conception of their role as advocates and a less clearly formulated notion of an intervener role. However, they expect to learn how to support speech and language development during intervention; (ii) parents' conception of role can change during involvement in speech and language therapy. Many parents' accounts refer to learning, reflecting and behaving differently with their child.

A number of previous studies indicate that parents expect to have an advocacy role, often including elements of assessment in order to judge when to seek help (Glogowska and Campbell, 2004; Law and Roy, 2008; Lindsay and Dockrell, 2004; McAllister et al., 2011). Other studies have shown parents' acceptance of 'observer' and 'helper' roles in intervention (Bowen and Cupples, 2004; Watt Pappas and McLeod, 2009; Watt Pappas et al 2016), but few refer to parents' views of learning to adopt a new role as intervener. The current study found that some parents described 
marked changes in their conception, adopting a new conception of role as intervener. This was expressed as either a sudden realisation or a growing awareness of the need to assume more involved roles. On occasions parents expressed a complete change in their approach. Parents conveyed a belief that they were thinking differently, rather than simply doing different activities, such as homework assigned by the professional. This is consistent with conceptual change theory (Vosniadou, 2013). Conceptual change aims to encourage a deep and lasting understanding for the learner based on restructuring learners' existing concepts. The examples in this study provide powerful exemplars of 'collaborative conceptual change' seen in the context of adult learning (Miyake 2013). For instance, some parents reflected on their learning during intervention as transformative, articulated as not only thinking differently about their child, but approaching parenting more positively.

The study indicates that parents often expect to be learners, even from the outset of involvement with the SLT. Previous research suggests that parents' interest in learning may not always be acknowledged by the roles that SLTs assign to them (Marshall et al., 2007; Watts Pappas and McLeod, 2009). This may be compounded by parents' own uncertainty about adopting an intervener role (Watts-Pappas et al., 2016). 
The findings have important clinical implications for intervention. First, in order to initiate a co-working partnership, SLTs may need to explicitly negotiate roles before intervention begins, encouraging parents to conceive of their role as an intervener. The data illustrates how some parents were uncertain about this role, but were ready to learn, and expressed marked changes in their conception as intervention progressed, similar to the conceptual change described in educational studies. Parents articulated doing different things with their child, but also doing things differently, which they referred to as a changed approach to parenting.

Secondly, parent education is becoming routine practice in pre-school speech and language therapy in England (Roulstone et al., 2012), but the professional discourse of adopting a teaching role is less frequently discussed. Teaching or coaching roles could complement SLT roles of assessment and intervention, drawing on conceptual change theory to encourage longer term changes in parents' understanding of themselves as interveners.

The study has three limitations. First, the sample size was relatively small and may have been influenced by the nature of the services provided. Purposive sampling helps to reduce this risk, and in this study, parents from a range of socio-economic backgrounds were included through purposive sampling. Nevertheless, the 
recruitment to the study may have been affected by the opt-in system that three services used, as, parents were already 'engaged' enough to arrange an appointment with the SLT via telephone. This study did not attempt to collect evidence from parents who did not opt into speech and language therapy services. Second, there was a risk of participation bias as recruitment depended on individuals volunteering to participate in the study and thus may have attracted parents who felt more interested or confident in expressing their views. Nevertheless, the opt-in rate to participate was relatively high and few parents refused to participate when they were approached. Thirdly, social desirability bias (King and Bruner, 2000) can influence the responses that participants make during data collection in interviews. This is characterised by participants responding in a manner they believe is more socially acceptable. This risk was reduced through using semi-structured interviews that allowed in-depth exploration of participants' views.

\section{Conclusions}

Parents have firm conceptions of their roles as advocates for their children but are less certain about adopting an intervener role when their child shows difficulties developing speech and language. Parents anticipate learning how to help their children when they attend speech and language therapy and evidence from this study 
indicates that some parents' conception of role changes as they work alongside the SLT. SLTs can make an important contribution within this partnership by enabling parents to assume new roles as interveners, acting as primary agents of change in supporting their child's speech and language development. In order to achieve this, SLTs may also need to consider how their own role can extend beyond assessment and intervention, explicitly assuming a teaching/coaching role to build parents roles as interveners.

\section{Acknowledgements}

This paper presents independent research funded by the National Institute for Health Research (NIHR) under its Programme Grants for Applied Research (Grant Reference Number RP-PG-0109-10073). The views expressed are those of the author(s) and not necessarily those of the NHS, the NIHR or the Department of Health.

\section{References}

Attride-Stirling J (2001) Thematic networks: an analytic tool for qualitative research. Qualitative Research 1: 385-405.

Bowen C and Cupples L (2004) The role of families in optimizing phonological therapy outcomes. Child Language Teaching and Therapy 20: 245-260. 
Breakwell GM (2006) Interviewing methods. Research methods in psychology 3: 232253.

Bronfenbrenner U and Morris PA (2006) The bioecological model of human development. Handbook of child psychology.

Carroll C (2010) "It's not everyday that parents get a chance to talk like this": Exploring parents' perceptions and expectations of speech-language pathology services for children with intellectual disability. International Journal of SpeechLanguage Pathology 12: 352-361.

Cunningham BJ and Rosenbaum PL (2015) A Bioecological framework to evaluate communicative participation outcomes for preschoolers receiving speech-language therapy interventions in Ontario, Canada. International Journal of Language \& Communication Disorders 50: 405-415.

Department for Education and Department for Health (2015) Special educational needs and disability code of practice. 0-25 years. London. Department for Education. Glogowska M and Campbell R (2004) Parental Views of Surveillance for Early Speech and Language Difficulties. Children and Society 18:266-277.

Goodall J and Montgomery C (2014) Parental involvement to parental engagement: a continuum. Educational Review 66: 399-410 
Goodall J and Vorhaus J (2011) Review of best practice in parental engagement. London: Department for Education.

Kaufmann DR, Keselman A, Patel VL. (2013) Conceptual understanding in the domain of health in (Ed) Vosniadou, S. International handbook of research on conceptual change. 2nd Edition. New York: Routledge.

King MF, and Bruner GC. (2000) 'Social Desirability Bias: A Neglected Aspect of Validity Testing.' Psychology and Marketing 17: 79-103.

Klatte I, Roulestone S. (2016) The practical side of working with parent-child interaction therapy with pre-school childen with language impairments. Child Language Teaching and Therapy: 1-15.

Law J Garrett Z and Nye C (2008) Speech and language therapy interventions for children with primary speech and language delay or disorder. Cochrane Collabaration 1:1-62.

Law J and Roy P (2008) Parental Report of Infant Language Skills: A Review of the Development and Application of the Communicative Development Inventories. Child and Adolescent Mental Health 13: 198-206.

Lindsay G and Dockrell JE (2004) Whose job is it? Parents' concerns about the needs of their children with language problems. Journal of Special Education 37: 225. 
McAllister L, McCormack J and McLeod (2011) Expectations and experiences of accessing and participating in services for childhood speech impairment. International Journal of Speech - Language Pathology 13: 251-267.

Marshall J, Goldbart J and Philips J (2007) Parents' and speech and language therapists' explanatory models of language development, language delay and intervention. International Journal of Language and Communication Disorders 42: 533555.

Miyake N (2013) Conceptual change through collaboration. In Vosniadou, S. (Ed.) International Handbook of Research on Conceptual Change. New York: Routledge.

Pintrich PR, Marx RW and Boyle RA (1993) Beyond Cold Conceptual Change: The Role of Motivational Beliefs and Classroom Contextual Factors in the Process of Conceptual Change. Review of Educational Research 63: 167-199.

Pring T, Dodd B and Joffe V (2012) The working practices and clinical experiences of paediatric speech and language therapists: a national UK survey. International Journal of Language and Communication Disorders 47: 696-708.

QSR International Pty Ltd (2012) NVIVO qualitative data analysis software v10. Melbourne. QSR International Pty Ltd. 
Ritchie J, Lewis J, Nicholls, CM and Ormston R (2013) Eds. Qualitative research practice: A guide for social science students and researchers. Sage

Ritchie J and Spencer L (2002) Qualitative data analysis for applied policy research. The qualitative researcher's companion. 573: 305-329.

Roberts M Y and Kaiser A P (2011) The effectiveness of parent-implemented language interventions: A meta-analysis. American Journal of Speech-Language Pathology 20: 180-199.

Roulstone S, Wren Y, Bakopoulou I, Goodlad S and Lindsay G (2012) Exploring interventions for children and young people with speech, language and communication needs: A study of practice. DFE-RR247-BCRP13, London: Department for Education. Vosniadou, S. (2007) 'Conceptual Change and Education. Human Development. 50: 4754.

Vosniadou, S. (2013) International handbook of research on conceptual change. 2nd Edition New York: Routledge.

Washington KN, Thomas-Stonell N, McLeod, S. (2012). Parents' perspective on the professional-child relationship and children's functional communication following speechlanguage intervention. Canadian Journal of Speech-Language Pathology and Audiology 36:226233 
Watts Pappas N, MacAllister L, McLeod S. (2016) Parental beliefs and experiences regarding involvement in intervention for their child with speech sound disorder. Child Language Teaching and Therapy: 223-239

Watts Pappas N and McLeod S (Eds.) (2009). Working with families in speech-language pathology. San Diego Oxford, Brisbane: Plural.

Watts Pappas NW, McLeod S McAllister L and Mcinnon D H (2008) Parental involvement in speech intervention: a national survey. Clinical Linguistics and Phonetics 22: 335 -344.

World Health Organisation (2007) International classification of functioning, disability and health: children and youth version: ICF-CY 2007, Geneva. WHO

\section{Appendix 1}

Topic guide for first set of interviews with parents

I'd like to talk to you about your experience of coming to speech and language therapy with your child. Could we talk about your journey in finding out that your child has some difficulties with learning to talk and what helped you?

1. Talk me through how your child first came to see the SLT

a. When did you realise he/she might need support 
b. Looking back is there anything you would change about what happened?

2. How were child's difficulties described or labelled

a. Before you came to SLT

b. After assessment with the SLT

c. Is there anything you would have changed about what happened?

3. Tell me about how decisions about what your child's needed were made. Could this have been done differently?

4. What kind of support is your child going to receive from the SLT. How will this be provided? Is there anything you would have changed about this?

5. At the beginning, what are you/did you hope for?. What did you expect from SLT? Were these expectations fulfilled (in what ways?)

6. How would you sum up your role in relation to your child and SLT?

7. In supporting your child's speech and language development, has your role changed over time? How do you thik it will change in the future?

8. How do you think your attendance here will affect your child's progress?

9. What do you think are the most important factors helping your child's progress? 
